\title{
A Korpelevich-like algorithm for variational inequalities
}

\author{
Zhitao $\mathrm{Wu}^{1}$, Yonghong $\mathrm{YaO}^{1 *}$, Yeong-Cheng Liou ${ }^{2}$ and Hong-Jun $\mathrm{Li}^{1}$
}

"Correspondence:

yaoyonghong@yahoo.cn

'Department of Mathematics, Tianjin Polytechnic University,

Tianjin, 300387, China

Full list of author information is

available at the end of the article

\begin{abstract}
A Korpelevich-like algorithm has been introduced for solving a generalized variational inequality. It is shown that the presented algorithm converges strongly to a special solution of the generalized variational inequality.

MSC: $47 \mathrm{H} 05 ; 47 J 25$

Keywords: Korpelevich-like algorithm; sunny nonexpansive retraction; generalized variational inequalities; $\alpha$-inverse-strongly accretive mappings; Banach spaces
\end{abstract}

\section{Introduction}

Now it is well-known that the variational inequality of finding $x^{\prime \prime} \in C$ such that

$$
\left\langle A x^{*}, x-x^{*}\right| \geq 0, \quad \forall x \in C,
$$

where $C$ is a nonempty closed convex subset of a real Hilbert space $H$ and $A: C \rightarrow H$ is a given mapping, is a fundamental problem in variational analysis and, in particular, in optimization theory. For related works, please see [1-20] and the references contained therein. Especially, Yao, Marino and Muglia [21] presented the following modified Korpelevich method for solving (1.1):

$$
\begin{aligned}
& y_{n}=P_{C}\left[x_{n}-\lambda A x_{n}-\alpha_{n} x_{n}\right], \\
& x_{n+1}=P_{C}\left[x_{n}-\lambda A y_{n}+\mu\left(y_{n}-x_{n}\right)\right], \quad n \geq 0 .
\end{aligned}
$$

Recently, Aoyama, Iiduka and Takahashi [22] extended the variational inequality (1.1) to Banach spaces as follows:

$$
\text { Find } x^{*} \in C \text { such that }\left\langle A x^{*}, J\left(x-x^{*}\right)\right\rangle \geq 0, \quad \forall x \in C,
$$

where $C$ is a nonempty closed convex subset of a real Banach space $E$. We use $S(C, A)$ to denote the solution set of (1.3). The generalized variational inequality (1.3) is connected with the fixed point problem for nonlinear mappings. For solving the above generalized variational inequality (1.3), Aoyama, Iiduka and Takahashi [22] introduced the iterative algorithm

$$
x_{n+1}=\alpha_{n} x_{n}+\left(1-\alpha_{n}\right) Q_{C}\left[x_{n}-\lambda_{n} A x_{n}\right], \quad n \geq 0,
$$

\section{Springer}

๑ 2013 Wu et al.; licensee Springer. This is an Open Access article distributed under the terms of the Creative Commons Attribution License (http://creativecommons.org/licenses/by/2.0), which permits unrestricted use, distribution, and reproduction in any medium, provided the original work is properly cited. 
where $Q_{C}$ is a sunny nonexpansive retraction from $E$ onto $C$ and $\left\{\alpha_{n}\right\} \subset(0,1),\left\{\lambda_{n}\right\} \subset$ $(0, \infty)$ are two real number sequences. Motivated by (1.4), Yao and Maruster [23] presented a modification of (1.4) as follows:

$$
x_{n+1}=\beta_{n} x_{n}+\left(1-\beta_{n}\right) Q_{C}\left[\left(1-\alpha_{n}\right)\left(x_{n}-\lambda A x_{n}\right)\right], \quad n \geq 0 .
$$

Motivated and inspired by the above algorithms (1.2), (1.4) and (1.5), in this paper, we suggest an extragradient-type method via the sunny nonexpansive retraction for solving the variational inequalities (1.3) in Banach spaces. It is shown that the presented algorithm converges strongly to a special solution of the variational inequality (1.3).

\section{Preliminaries}

Let $C$ be a nonempty closed convex subset of a real Banach space $E$. Recall that a mapping $A$ of $C$ into $E$ is said to be accretive if there exists $j(x-y) \in J(x-y)$ such that

$$
\langle A x-A y, j(x-y)\rangle \geq 0
$$

for all $x, y \in C$. A mapping $A$ of $C$ into $E$ is said to be $\alpha$-strongly accretive if for $\alpha>0$,

$$
\langle A x-A y, j(x-y)| \geq \alpha\|x-y\|^{2}
$$

for all $x, y \in C$. A mapping $A$ of $C$ into $E$ is said to be $\alpha$-inverse-strongly accretive if for $\alpha>0$,

$$
\langle A x-A y, j(x-y)| \geq \alpha\|A x-A y\|^{2}
$$

for all $x, y \in C$.

Let $U=\{x \in E:\|x\|=1\}$. A Banach space $E$ is said to uniformly convex if for each $\epsilon \epsilon$ $(0,2]$, there exists $\delta>0$ such that for any $x, y \in U$,

$$
\|x-y\| \geq \epsilon \quad \text { implies } \quad\left\|\frac{x+y}{2}\right\| \leq 1-\delta .
$$

It is known that a uniformly convex Banach space is reflexive and strictly convex. A Banach space $E$ is said to be smooth if the limit

$$
\lim _{t \rightarrow 0} \frac{\|x+t y\|-\|x\|}{t}
$$

exists for all $x, y \in U$. It is also said to be uniformly smooth if the limit (2.1) is attained uniformly for $x, y \in U$. The norm of $E$ is said to be Frechet differentiable if for each $x \in U$, the limit (2.1) is attained uniformly for $y \in U$. And we define a function $\rho:[0, \infty) \rightarrow[0, \infty)$ called the modulus of smoothness of $E$ as follows:

$$
\rho(\tau)=\sup \left\{\frac{1}{2}(\|x+y\|+\|x-y\|)-1: x, y \in X,\|x\|=1,\|y\|=\tau\right\} .
$$

It is known that $E$ is uniformly smooth if and only if $\lim _{\tau \rightarrow 0} \rho(\tau) / \tau=0$. Let $q$ be a fixed real number with $1<q \leq 2$. Then a Banach space $E$ is said to be $q$-uniformly smooth if there exists a constant $c>0$ such that $\rho(\tau) \leq c \tau^{q}$ for all $\tau>0$.

We need the following lemmas for the proof of our main results. 
Lemma 2.1 [24] Let $q$ be a given real number with $1<q \leq 2$ and let $E$ be a $q$-uniformly smooth Banach space. Then

$$
\left.\|x+y\|^{q} \leq\|x\|^{q}+q \mid y, J_{q}(x)\right)+2\|K y\|^{q}
$$

for all $x, y \in E$, where $K$ is the $q$-uniformly smoothness constant of $E$ and $J_{q}$ is the generalized duality mapping from $E$ into $2^{E^{*}}$ defined by

$$
J_{q}(x)=\left\{f \in E^{*}:\langle x, f\rangle=\|x\|^{q},\|f\|=\|x\|^{q-1}\right\}, \quad \forall x \in E .
$$

Let $D$ be a subset of $C$ and let $Q$ be a mapping of $C$ into $D$. Then $Q$ is said to be sunny if

$$
Q(Q x+t(x-Q x))=Q x,
$$

whenever $Q x+t(x-Q x) \in C$ for $x \in C$ and $t \geq 0$. A mapping $Q$ of $C$ into itself is called a retraction if $Q^{2}=Q$. If a mapping $Q$ of $C$ into itself is a retraction, then $Q z=z$ for every $z \in R(Q)$, where $R(Q)$ is the range of $Q$. A subset $D$ of $C$ is called a sunny nonexpansive retract of $C$ if there exists a sunny nonexpansive retraction from $C$ onto $D$. We know the following lemma concerning sunny nonexpansive retraction.

Lemma 2.2 [25] Let $C$ be a closed convex subset of a smooth Banach space E, let D be a nonempty subset of $C$ and $Q$ be a retraction from $C$ onto $D$. Then $Q$ is sunny and nonexpansive if and only if

$$
\langle u-Q u, j(y-Q u)\rangle \leq 0
$$

for all $u \in C$ and $y \in D$.

Lemma 2.3 [22] Let C be a nonempty closed convex subset of a smooth Banach space X. Let $Q_{C}$ be a sunny nonexpansive retraction from $X$ onto $C$ and let $A$ be an accretive operator of $C$ into $X$. Then for all $\lambda>0$,

$$
S(C, A)=F\left(Q_{C}(I-\lambda A)\right),
$$

where $S(C, A)=\left\{x^{*} \in C:\left\langle A x^{*}, J\left(x-x^{*}\right)\right\rangle \geq 0, \forall x \in C\right\}$.

Lemma 2.4 [26] Let C be a nonempty closed convex subset of a real 2-uniformly smooth Banach space $X$. Let the mapping $A: C \rightarrow X$ be $\alpha$-inverse-strongly accretive. Then we have

$$
\|(I-\lambda A) x-(I-\lambda A) y\|^{2} \leq\|x-y\|^{2}+2 \lambda\left(K^{2} \lambda-\alpha\right)\|A x-A y\|^{2} .
$$

In particular, if $0 \leq \lambda \leq \frac{\alpha}{K^{2}}$, then $I-\lambda A$ is nonexpansive.

Proof Indeed, for all $x, y \in C$, from Lemma 2.1, we have

$$
\begin{aligned}
\|(I-\lambda A) x-(I-\lambda A) y\|^{2} & =\|(x-y)-\lambda(A x-A y)\|^{2} \\
& \leq\|x-y\|^{2}-2 \lambda\langle A x-A y, j(x-y)\rangle+2 K^{2} \lambda^{2}\|A x-A y\|^{2}
\end{aligned}
$$




$$
\begin{aligned}
& \leq\|x-y\|^{2}-2 \lambda \alpha\|A x-A y\|^{2}+2 K^{2} \lambda^{2}\|A x-A y\|^{2} \\
& =\|x-y\|^{2}+2 \lambda\left(K^{2} \lambda-\alpha\right)\|A x-A y\|^{2} .
\end{aligned}
$$

It is clear that if $0 \leq \lambda \leq \frac{\alpha}{K^{2}}$, then $I-\lambda A$ is nonexpansive.

Lemma 2.5 [27] Let $C$ be a nonempty bounded closed convex subset of a uniformly convex Banach space $E$ and let $T$ be a nonexpansive mapping of $C$ into itself. If $\left\{x_{n}\right\}$ is a sequence of $C$ such that $x_{n} \rightarrow x$ weakly and $x_{n}-T x_{n} \rightarrow 0$ strongly, then $x$ is a fixed point of $T$.

Lemma 2.6 [28] Assume $\left\{a_{n}\right\}$ is a sequence of nonnegative real numbers such that

$$
a_{n+1} \leq\left(1-\gamma_{n}\right) a_{n}+\delta_{n}, \quad n \geq 0,
$$

where $\left\{\gamma_{n}\right\}$ is a sequence in $(0,1)$ and $\left\{\delta_{n}\right\}$ is a sequence in $R$ such that

(a) $\sum_{n=0}^{\infty} \gamma_{n}=\infty$;

(b) $\lim \sup _{n \rightarrow \infty} \delta_{n} / \gamma_{n} \leq 0$ or $\sum_{n=0}^{\infty}\left|\delta_{n}\right|<\infty$.

Then $\lim _{n \rightarrow \infty} a_{n}=0$.

\section{Main results}

In this section, we present our Korpelevich-like algorithm and consequently we will show its strong convergence.

\subsection{Conditions assumptions}

(A1) $E$ is a uniformly convex and 2-uniformly smooth Banach space with a weakly sequentially continuous duality mapping;

(A2) $C$ is a nonempty closed convex subset of $E$;

(A3) $A: C \rightarrow E$ is an $\alpha$-strongly accretive and $L$-Lipschitz continuous mapping with $S(C, A) \neq \emptyset$

(A4) $Q_{C}$ is a sunny nonexpansive retraction from $E$ onto $C$.

\subsection{Parameters restrictions}

(P1) $\lambda, \mu$ and $\gamma$ are three positive constants satisfying:

(i) $\gamma \in(0,1), \lambda \in[a, b]$ for some $a, b$ with $0<a<b<\frac{\alpha}{K^{2} L^{2}}$;

(ii) $\frac{\lambda}{\mu}<\frac{\alpha}{K^{2} L^{2}}$ where $K$ is the smooth constant of $E$.

(P2) $\left\{\alpha_{n}\right\}$ is a sequence in $(0,1)$ such that $\lim _{n \rightarrow \infty} \alpha_{n}=0$ and $\sum_{n=1}^{\infty} \alpha_{n}=\infty$.

Algorithm 3.1 For given $x_{0} \in C$, define a sequence $\left\{x_{n}\right\}$ iteratively by

$$
\left\{\begin{array}{l}
y_{n}=Q_{C}\left[\left(1-\alpha_{n}\right) x_{n}-\lambda A x_{n}\right], \\
x_{n+1}=(1-\gamma) x_{n}+\gamma Q_{C}\left[x_{n}-\lambda A y_{n}+\mu\left(y_{n}-x_{n}\right)\right], \quad n \geq 0 .
\end{array}\right.
$$

Theorem 3.2 The sequence $\left\{x_{n}\right\}$ generated by (3.1) converges strongly to $Q^{\prime}(0)$, where $Q^{\prime}$ is a sunny nonexpansive retraction of $E$ onto $S(C, A)$.

Proof Let $p \in S(C, A)$. First, from Lemma 2.2, we have $p=Q_{C}[p-\delta A p]$ for all $\delta>0$. In particular, $p=Q_{C}[p-\lambda A p]=Q_{C}\left[\alpha_{n} p+\left(1-\alpha_{n}\right)\left(p-\frac{\lambda}{1-\alpha_{n}} A p\right)\right]$ for all $n \geq 0$. 
Since $A: C \rightarrow E$ is $\alpha$-strongly accretive and $L$-Lipschitzian, it must be $\frac{\alpha}{L^{2}}$-inversestrongly accretive mapping. Thus, by Lemma 2.4 , we have

$$
\|(I-\lambda A) x-(I-\lambda A) y\|^{2} \leq\|x-y\|^{2}+2 \lambda\left(K^{2} \lambda-\frac{\alpha}{L^{2}}\right)\|A x-A y\|^{2} .
$$

Since $\alpha_{n} \rightarrow 0$ and $\lambda \in[a, b] \subset\left(0, \frac{\alpha}{K^{2} L^{2}}\right)$, we get $\alpha_{n}<1-\frac{K^{2} L^{2} \lambda}{\alpha}$ for enough large $n$. Without loss of generality, we may assume that for all $n \in \mathbb{N}, \alpha_{n}<1-\frac{K^{2} L^{2} \lambda}{\alpha}$, i.e., $\frac{\lambda}{1-\alpha_{n}} \in\left(0, \frac{\alpha}{K^{2} L^{2}}\right)$. Hence, $I-\frac{\lambda}{1-\alpha_{n}} A$ is nonexpansive.

From (3.1), we have

$$
\begin{aligned}
\left\|y_{n}-p\right\| & =\left\|Q_{C}\left[\left(1-\alpha_{n}\right) x_{n}-\lambda A x_{n}\right]-Q_{C}\left[\alpha_{n} p+\left(1-\alpha_{n}\right)\left(p-\frac{\lambda}{1-\alpha_{n}} A p\right)\right]\right\| \\
& \leq\left\|\alpha_{n}(-p)+\left(1-\alpha_{n}\right)\left[\left(x_{n}-\frac{\lambda}{1-\alpha_{n}} A x_{n}\right)-\left(p-\frac{\lambda}{1-\alpha_{n}} A p\right)\right]\right\| \\
& \leq \alpha_{n}\|p\|+\left(1-\alpha_{n}\right)\left\|\left(I-\frac{\lambda}{1-\alpha_{n}} A\right) x_{n}-\left(I-\frac{\lambda}{1-\alpha_{n}} A\right) p\right\| \\
& \leq \alpha_{n}\|p\|+\left(1-\alpha_{n}\right)\left\|x_{n}-p\right\| .
\end{aligned}
$$

By (3.1) and (3.2), we have

$$
\begin{aligned}
\left\|x_{n+1}-p\right\| \leq & (1-\gamma)\left\|x_{n}-p\right\|+\gamma\left\|Q_{C}\left[x_{n}-\lambda A y_{n}+\mu\left(y_{n}-x_{n}\right)\right]-p\right\| \\
= & (1-\gamma)\left\|x_{n}-p\right\|+\gamma \| Q_{C}\left[(1-\mu) x_{n}+\mu\left(y_{n}-\frac{\lambda}{\mu} A y_{n}\right)\right] \\
& -Q_{C}\left[(1-\mu) p+\mu\left(p-\frac{\lambda}{\mu} A p\right)\right] \| \\
\leq & (1-\gamma)\left\|x_{n}-p\right\| \\
& +\gamma\left\|(1-\mu)\left(x_{n}-p\right)+\mu\left[\left(y_{n}-\frac{\lambda}{\mu} A y_{n}\right)-\left(p-\frac{\lambda}{\mu} A p\right)\right]\right\| \\
\leq & (1-\gamma)\left\|x_{n}-p\right\|+(1-\mu) \gamma\left\|x_{n}-p\right\| \\
& +\mu \gamma\left\|\left(y_{n}-\frac{\lambda}{\mu} A y_{n}\right)-\left(p-\frac{\lambda}{\mu} A p\right)\right\| \\
\leq & (1-\mu \gamma)\left\|x_{n}-p\right\|+\mu \gamma\left\|y_{n}-p\right\| \\
\leq & (1-\mu \gamma)\left\|x_{n}-p\right\|+\mu \gamma \alpha_{n}\|p\|+\mu \gamma\left(1-\alpha_{n}\right)\left\|x_{n}-p\right\| \\
= & \left(1-\mu \gamma \alpha_{n}\right)\left\|x_{n}-p\right\|+\mu \gamma \alpha_{n}\|p\| \\
\leq & \max \left\{\left\|x_{n}-p\right\|,\|p\|\right\} \\
\vdots & \\
\leq & \max \left\{\left\|x_{0}-p\right\|,\|p\|\right\} .
\end{aligned}
$$

Hence, $\left\{x_{n}\right\}$ is bounded. 
Set $z_{n}=Q_{C}\left[x_{n}-\lambda A y_{n}+\mu\left(y_{n}-x_{n}\right)\right]$. From (3.1), we have $x_{n+1}=(1-\gamma) x_{n}+\gamma z_{n}$ for all $n \geq 0$. Then we have

$$
\begin{aligned}
\left\|y_{n}-y_{n-1}\right\|= & \left\|Q_{C}\left[\left(1-\alpha_{n}\right) x_{n}-\lambda A x_{n}\right]-Q_{C}\left[\left(1-\alpha_{n-1}\right) x_{n-1}-\lambda A x_{n-1}\right]\right\| \\
\leq & \left\|\left(1-\alpha_{n}\right)\left(x_{n}-\frac{\lambda}{1-\alpha_{n}} A x_{n}\right)-\left(1-\alpha_{n-1}\right)\left(x_{n-1}-\frac{\lambda}{1-\alpha_{n-1}} A x_{n-1}\right)\right\| \\
\leq & \left(1-\alpha_{n}\right)\left\|\left(x_{n}-\frac{\lambda}{1-\alpha_{n}} A x_{n}\right)-\left(x_{n-1}-\frac{\lambda}{1-\alpha_{n}} A x_{n-1}\right)\right\| \\
& +\left|\alpha_{n}-\alpha_{n-1}\right|\left\|x_{n-1}\right\| \\
\leq & \left(1-\alpha_{n}\right)\left\|x_{n}-x_{n-1}\right\|+\left|\alpha_{n}-\alpha_{n-1}\right|\left\|x_{n-1}\right\|,
\end{aligned}
$$

and thus

$$
\begin{aligned}
\left\|z_{n}-z_{n-1}\right\| & =\left\|Q_{C}\left[x_{n}-\lambda A y_{n}+\mu\left(y_{n}-x_{n}\right)\right]-Q_{C}\left[x_{n-1}-\lambda A y_{n-1}+\mu\left(y_{n-1}-x_{n-1}\right)\right]\right\| \\
& \leq(1-\mu)\left\|x_{n}-x_{n-1}\right\|+\mu\left\|\left(y_{n}-\frac{\lambda}{\mu} A y_{n}\right)-\left(y_{n-1}-\frac{\lambda}{\mu} A y_{n-1}\right)\right\| \\
& \leq(1-\mu)\left\|x_{n}-x_{n-1}\right\|+\mu\left\|y_{n}-y_{n-1}\right\| \\
& \leq\left(1-\mu \alpha_{n}\right)\left\|x_{n}-x_{n-1}\right\|+\left|\alpha_{n}-\alpha_{n-1}\right|\left\|x_{n-1}\right\| .
\end{aligned}
$$

It follows that

$$
\limsup _{n \rightarrow \infty}\left(\left\|z_{n}-z_{n-1}\right\|-\left\|x_{n}-x_{n-1}\right\|\right) \leq 0 .
$$

This together with Lemma 2.6 implies that

$$
\lim _{n \rightarrow \infty}\left\|x_{n+1}-x_{n}\right\|=0 .
$$

From (3.2), we have

$$
\begin{aligned}
\left\|y_{n}-p\right\|^{2} & \leq\left\|\alpha_{n}(-p)+\left(1-\alpha_{n}\right)\left[\left(x_{n}-\frac{\lambda}{1-\alpha_{n}} A x_{n}\right)-\left(p-\frac{\lambda}{1-\alpha_{n}} A p\right)\right]\right\|^{2} \\
& \leq \alpha_{n}\|p\|^{2}+\left(1-\alpha_{n}\right)\left\|\left(x_{n}-\frac{\lambda}{1-\alpha_{n}} A x_{n}\right)-\left(p-\frac{\lambda}{1-\alpha_{n}} A p\right)\right\|^{2} \\
& \leq \alpha_{n}\|p\|^{2}+\left(1-\alpha_{n}\right)\left\|x_{n}-p\right\|^{2}+2 \lambda\left(\frac{K^{2} \lambda}{1-\alpha_{n}}-\frac{\alpha}{L^{2}}\right)\left\|A x_{n}-A p\right\|^{2} .
\end{aligned}
$$

From (3.1), (3.3) and (3.4), we obtain

$$
\begin{aligned}
& \left\|x_{n+1}-p\right\|^{2} \\
& \quad \leq(1-\gamma)\left\|x_{n}-p\right\|^{2}+\gamma\left\|(1-\mu)\left(x_{n}-p\right)+\mu\left[\left(y_{n}-\frac{\lambda}{\mu} A y_{n}\right)-\left(p-\frac{\lambda}{\mu} A p\right)\right]\right\|^{2} \\
& \quad \leq(1-\gamma)\left\|x_{n}-p\right\|^{2}+\gamma(1-\mu)\left\|x_{n}-p\right\|^{2}+\gamma \mu\left\|\left(y_{n}-\frac{\lambda}{\mu} A y_{n}\right)-\left(p-\frac{\lambda}{\mu} A p\right)\right\|^{2}
\end{aligned}
$$




$$
\begin{aligned}
\leq & (1-\gamma \mu)\left\|x_{n}-p\right\|^{2}+\gamma \mu\left[\left\|y_{n}-p\right\|^{2}+\frac{2 \lambda}{\mu}\left(\frac{K^{2} \lambda}{\mu}-\frac{\alpha}{L^{2}}\right)\left\|A y_{n}-A p\right\|^{2}\right] \\
\leq & \gamma \mu\left[\alpha_{n}\|p\|^{2}+\left(1-\alpha_{n}\right)\left\|x_{n}-p\right\|^{2}+2 \lambda\left(\frac{K^{2} \lambda}{1-\alpha_{n}}-\frac{\alpha}{L^{2}}\right)\left\|A x_{n}-A p\right\|^{2}\right] \\
& +(1-\gamma \mu)\left\|x_{n}-p\right\|^{2}+2 \gamma \lambda\left(\frac{K^{2} \lambda}{\mu}-\frac{\alpha}{L^{2}}\right)\left\|A y_{n}-A p\right\|^{2} \\
= & \alpha_{n} \gamma \mu\|p\|^{2}+\left(1-\gamma \mu \alpha_{n}\right)\left\|x_{n}-p\right\|^{2}+2 \gamma \lambda \mu\left(\frac{K^{2} \lambda}{1-\alpha_{n}}-\frac{\alpha}{L^{2}}\right)\left\|A x_{n}-A p\right\|^{2} \\
& +2 \gamma \lambda \mu\left(\frac{K^{2} \lambda}{\mu}-\frac{\alpha}{L^{2}}\right)\left\|A y_{n}-A p\right\|^{2} .
\end{aligned}
$$

Therefore, we have

$$
\begin{aligned}
0 & \leq-2 \gamma \lambda \mu\left(\frac{K^{2} \lambda}{1-\alpha_{n}}-\frac{\alpha}{L^{2}}\right)\left\|A x_{n}-A p\right\|^{2}-2 \gamma \lambda \mu\left(\frac{K^{2} \lambda}{\mu}-\frac{\alpha}{L^{2}}\right)\left\|A y_{n}-A p\right\|^{2} \\
& \leq \alpha_{n} \gamma \mu\|p\|^{2}+\left\|x_{n}-p\right\|^{2}-\left\|x_{n+1}-p\right\|^{2} \\
& =\alpha_{n} \gamma \mu\|p\|^{2}+\left(\left\|x_{n}-p\right\|+\left\|x_{n+1}-p\right\|\right)\left(\left\|x_{n}-p\right\|-\left\|x_{n+1}-p\right\|\right) \\
& \leq \alpha_{n} \gamma \mu\|p\|^{2}+\left(\left\|x_{n}-p\right\|+\left\|x_{n+1}-p\right\|\right)\left\|x_{n}-x_{n+1}\right\| .
\end{aligned}
$$

Since $\alpha_{n} \rightarrow 0$ and $\left\|x_{n}-x_{n+1}\right\| \rightarrow 0$, we obtain

$$
\lim _{n \rightarrow \infty}\left\|A x_{n}-A p\right\|=\lim _{n \rightarrow \infty}\left\|A y_{n}-A p\right\|=0 .
$$

It follows that

$$
\lim _{n \rightarrow \infty}\left\|A y_{n}-A x_{n}\right\|=0
$$

Since $A$ is $\alpha$-strongly accretive, we deduce

$$
\left\|A y_{n}-A x_{n}\right\| \geq \alpha\left\|y_{n}-x_{n}\right\|,
$$

which implies that

$$
\lim _{n \rightarrow \infty}\left\|y_{n}-x_{n}\right\|=0
$$

that is,

$$
\lim _{n \rightarrow \infty}\left\|Q_{C}\left[\left(1-\alpha_{n}\right) x_{n}-\lambda A x_{n}\right]-x_{n}\right\|=0 .
$$

It follows that

$$
\lim _{n \rightarrow \infty}\left\|Q_{C}\left[x_{n}-\lambda A x_{n}\right]-x_{n}\right\|=0 .
$$

Next, we show that

$$
\limsup _{n \rightarrow \infty}\left\langle Q^{\prime}(0), j\left(x_{n}-Q^{\prime}(0)\right)\right\rangle \geq 0 .
$$


To show (3.6), since $\left\{x_{n}\right\}$ is bounded, we can choose a sequence $\left\{x_{n_{i}}\right\}$ of $\left\{x_{n}\right\}$ converging weakly to $z$ such that

$$
\limsup _{n \rightarrow \infty}\left\langle Q^{\prime}(0), j\left(x_{n}-Q^{\prime}(0)\right)\right\rangle=\limsup _{i \rightarrow \infty}\left\langle Q^{\prime}(0), j\left(x_{n_{i}}-Q^{\prime}(0)\right)\right\rangle
$$

We first prove $z \in S(C, A)$. It follows that

$$
\lim _{i \rightarrow \infty}\left\|Q_{C}(I-\lambda A) x_{n_{i}}-x_{n_{i}}\right\|=0
$$

By Lemma 2.5 and (3.8), we have $z \in F\left(Q_{C}(I-\lambda A)\right)$, it follows from Lemma 2.3 that $z \in$ $S(C, A)$.

Now, from (3.7) and Lemma 2.2, we have

$$
\begin{aligned}
\limsup _{n \rightarrow \infty}\left\langle Q^{\prime}(0), j\left(x_{n}-Q^{\prime}(0)\right)\right\rangle & =\limsup _{i \rightarrow \infty}\left\langle Q^{\prime}(0), j\left(x_{n_{i}}-Q^{\prime}(0)\right)\right\rangle \\
& =\left\langle Q^{\prime}(0), j\left(z-Q^{\prime}(0)\right)\right\rangle \\
& \geq 0
\end{aligned}
$$

Noticing that $\left\|x_{n}-y_{n}\right\| \rightarrow 0$, we deduce that

$$
\limsup _{n \rightarrow \infty}\left\langle Q^{\prime}(0), j\left(y_{n}-Q^{\prime}(0)\right)\right\rangle \geq 0
$$

Since $y_{n}=Q_{C}\left[\left(1-\alpha_{n}\right)\left(x_{n}-\frac{\lambda}{1-\alpha_{n}} A x_{n}\right)\right]$ and $Q^{\prime}(0)=Q_{C}\left[\alpha_{n} Q^{\prime}(0)+\left(1-\alpha_{n}\right)\left(Q^{\prime}(0)-\frac{\lambda}{1-\alpha_{n}} A Q^{\prime}(0)\right)\right]$ for all $n \geq 0$, we can deduce from Lemma 2.2 that

$$
\left\langle Q_{C}\left[\left(1-\alpha_{n}\right)\left(x_{n}-\frac{\lambda}{1-\alpha_{n}} A x_{n}\right)\right]-\left[\left(1-\alpha_{n}\right)\left(x_{n}-\frac{\lambda}{1-\alpha_{n}} A x_{n}\right)\right], j\left(y_{n}-Q^{\prime}(0)\right)\right\rangle \leq 0
$$

and

$$
\begin{aligned}
& \left\langle\left[\alpha_{n} Q^{\prime}(0)+\left(1-\alpha_{n}\right)\left(Q^{\prime}(0)-\frac{\lambda}{1-\alpha_{n}} A Q^{\prime}(0)\right)\right]\right. \\
& \left.\quad-Q_{C}\left[\alpha_{n} Q^{\prime}(0)+\left(1-\alpha_{n}\right)\left(Q^{\prime}(0)-\frac{\lambda}{1-\alpha_{n}} A Q^{\prime}(0)\right)\right], j\left(y_{n}-Q^{\prime}(0)\right)\right\rangle \leq 0 .
\end{aligned}
$$

Therefore, we have

$$
\begin{aligned}
\left\|y_{n}-Q^{\prime}(0)\right\|^{2} & \| Q_{C}\left[\left(1-\alpha_{n}\right)\left(x_{n}-\frac{\lambda}{1-\alpha_{n}} A x_{n}\right)\right] \\
& -Q_{C}\left[\alpha_{n} Q^{\prime}(0)+\left(1-\alpha_{n}\right)\left(Q^{\prime}(0)-\frac{\lambda}{1-\alpha_{n}} A Q^{\prime}(0)\right)\right] \|^{2} \\
\leq & \left\langle\alpha_{n}\left(-Q^{\prime}(0)\right)+\left(1-\alpha_{n}\right)\left[\left(x_{n}-\frac{\lambda}{1-\alpha_{n}} A x_{n}\right)\right.\right. \\
& \left.\left.-\left(Q^{\prime}(0)-\frac{\lambda}{1-\alpha_{n}} A Q^{\prime}(0)\right)\right], j\left(y_{n}-Q^{\prime}(0)\right)\right\rangle
\end{aligned}
$$




$$
\begin{aligned}
\leq & -\alpha_{n}\left\langle Q^{\prime}(0), j\left(y_{n}-Q^{\prime}(0)\right)\right\rangle+\left(1-\alpha_{n}\right) \|\left(x_{n}-\frac{\lambda}{1-\alpha_{n}} A x_{n}\right) \\
& -\left(Q^{\prime}(0)-\frac{\lambda}{1-\alpha_{n}} A Q^{\prime}(0)\right)\|\| y_{n}-Q^{\prime}(0) \| \\
\leq & -\alpha_{n}\left\langle Q^{\prime}(0), j\left(y_{n}-Q^{\prime}(0)\right)\right\rangle+\left(1-\alpha_{n}\right)\left\|x_{n}-Q^{\prime}(0)\right\|\left\|y_{n}-Q^{\prime}(0)\right\| \\
\leq & -\alpha_{n}\left\langle Q^{\prime}(0), j\left(y_{n}-Q^{\prime}(0)\right)\right\rangle+\frac{1-\alpha_{n}}{2}\left(\left\|x_{n}-Q^{\prime}(0)\right\|^{2}+\left\|y_{n}-Q^{\prime}(0)\right\|^{2}\right),
\end{aligned}
$$

which implies that

$$
\left\|y_{n}-Q^{\prime}(0)\right\|^{2} \leq\left(1-\alpha_{n}\right)\left\|x_{n}-Q^{\prime}(0)\right\|^{2}+2 \alpha_{n}\left(-Q^{\prime}(0), j\left(y_{n}-Q^{\prime}(0)\right)\right\rangle
$$

Finally, we will prove that the sequence $x_{n} \rightarrow Q^{\prime}(0)$. As a matter of fact, from (3.1) and (3.9), we have

$$
\begin{aligned}
&\left\|x_{n+1}-Q^{\prime}(0)\right\|^{2} \\
& \leq(1-\gamma)\left\|x_{n}-Q^{\prime}(0)\right\|^{2} \\
& \quad+\gamma\left\|(1-\mu)\left(x_{n}-Q^{\prime}(0)\right)+\mu\left[\left(y_{n}-\frac{\lambda}{\mu} A y_{n}\right)-\left(Q^{\prime}(0)-\frac{\lambda}{\mu} A Q^{\prime}(0)\right)\right]\right\|^{2} \\
& \leq(1-\gamma \mu)\left\|x_{n}-Q^{\prime}(0)\right\|^{2}+\gamma \mu\left\|\left(y_{n}-\frac{\lambda}{\mu} A y_{n}\right)-\left(Q^{\prime}(0)-\frac{\lambda}{\mu} A Q^{\prime}(0)\right)\right\|^{2} \\
& \leq(1-\gamma \mu)\left\|x_{n}-Q^{\prime}(0)\right\|^{2}+\gamma \mu\left\|y_{n}-Q^{\prime}(0)\right\|^{2} \\
& \leq\left(1-\gamma \mu \alpha_{n}\right)\left\|x_{n}-Q^{\prime}(0)\right\|^{2}+2 \gamma \mu \alpha_{n}\left(-Q^{\prime}(0), j\left(y_{n}-Q^{\prime}(0)\right)\right\rangle .
\end{aligned}
$$

Applying Lemma 2.6 to the last inequality, we conclude that $x_{n}$ converges strongly to $Q^{\prime}(0)$. This completes the proof.

\section{Competing interests}

The authors declare that they have no competing interests.

\section{Authors' contributions}

All authors contributed equally and significantly in writing this paper. All authors read and approved the final manuscript.

\section{Author details}

'Department of Mathematics, Tianjin Polytechnic University, Tianjin, 300387, China. ${ }^{2}$ Department of Information Management, Cheng Shiu University, Kaohsiung, 833, Taiwan.

\section{Acknowledgements}

Yonghong Yao was supported in part by NSFC 11071279 and NSFC 71161001-G0105. Yeong-Cheng Liou was supported in part by NSC 101-2628-E-230-001-MY3 and NSC 101-2622-E-230-005-CC3.

Received: 6 October 2012 Accepted: 4 February 2013 Published: 28 February 2013

\section{References}

1. Korpelevich, GM: An extragradient method for finding saddle points and for other problems. Ekon. Mat. Metod. 12, 747-756 (1976)

2. lusem, AN, Svaiter, BF: A variant of Korpelevich's method for variational inequalities with a new search strategy. Optimization 42, 309-321 (1997)

3. Iusem, AN, Lucambio Peŕez, LR: An extragradient-type algorithm for non-smooth variational inequalities. Optimization 48, 309-332 (2000)

4. Solodov, MV, Tseng, P: Modified projection-type methods for monotone variational inequalities. SIAM J. Control Optim. 34, 1814-1830 (1996) 
5. Lions, JL, Stampacchia, G: Variational inequalities. Commun. Pure Appl. Math. 20, 493-517 (1967)

6. He, BS, Yang, ZH, Yuan, XM: An approximate proximal-extragradient type method for monotone variational inequalities. J. Math. Anal. Appl. 300, 362-374 (2004)

7. Bello Cruz, JY, lusem, AN: A strongly convergent direct method for monotone variational inequalities in Hilbert space. Numer. Funct. Anal. Optim. 30(1-2), 23-36 (2009)

8. Glowinski, R: Numerical Methods for Nonlinear Variational Problems. Springer, New York (1984)

9. Yao, Y, Noor, MA, Liou, YC: Strong convergence of a modified extra-gradient method to the minimum-norm solution of variational inequalities. Abstr. Appl. Anal. 2012, Article ID 817436 (2012). doi:10.1155/2012/817436

10. $\mathrm{Xu}, \mathrm{HK}, \mathrm{Kim}, \mathrm{TH}$ : Convergence of hybrid steepest-descent methods for variational inequalities. J. Optim. Theory Appl. 119, 185-201 (2003)

11. Yamada, I: The hybrid steepest descent for the variational inequality problems over the intersection of fixed points sets of nonexpansive mappings. In: Butnariu, D, Censor, Y, Reich, S (eds.) Inherently Parallel Algorithms in Feasibility and Optimization and Their Applications, pp. 473-504. Elsevier, New York (2001)

12. Yao, Y, Liou, YC, Kang, SM: Two-step projection methods for a system of variational inequality problems in Banach spaces. J. Glob. Optim. 55, 801-811 (2013). doi:10.1007/s10898-011-9804-0

13. Yao, Y, Chen, R, Liou, YC: A unified implicit algorithm for solving the triple-hierarchical constrained optimization problem. Math. Comput. Model. 55, 1506-1515 (2012)

14. Yao, Y, Noor, MA, Noor, Kl, Liou, YC, Yaqoob, H: Modified extragradient method for a system of variational inequalities in Banach spaces. Acta Appl. Math. 110, 1211-1224 (2010)

15. Cho, YJ, Yao, Y, Zhou, H: Strong convergence of an iterative algorithm for accretive operators in Banach spaces. J. Comput. Anal. Appl. 10, 113-125 (2008)

16. Yao, Y, Cho, YJ, Liou, YC: Algorithms of common solutions for variational inclusions, mixed equilibrium problems and fixed point problems. Eur. J. Oper. Res. 212, 242-250 (2011)

17. Cho, YJ, Kang, SM, Qin, X: On systems of generalized nonlinear variational inequalities in Banach spaces. Appl. Math. Comput. 206, 214-220 (2008)

18. Cho, YJ, Qin, X: Systems of generalized nonlinear variational inequalities and its projection methods. Nonlinear Anal. 69, 4443-4451 (2008)

19. Ceng, LC, Ansari, QH, Yao, JC: Mann type steepest-descent and modified hybrid steepest-descent methods for variational inequalities in Banach spaces. Numer. Funct. Anal. Optim. 29, 987-1033 (2008)

20. Sahu, DR, Wong, NC, Yao, JC: A unified hybrid iterative method for solving variational inequalities involving generalized pseudo-contractive mappings. SIAM J. Control Optim. 50, 2335-2354 (2012)

21. Yao, Y, Marino, G, Muglia, L: A modified Korpelevich's method convergent to the minimum norm solution of a variational inequality. Optimization (in press). doi:10.1080/02331934.2013.764522

22. Aoyama, K, liduka, H, Takahashi, W: Weak convergence of an iterative sequence for accretive operators in Banach spaces. Fixed Point Theory Appl. 2006, Article ID 35390 (2006). doi:10.1155/FPTA/2006/35390

23. Yao, Y, Maruster, S: Strong convergence of an iterative algorithm for variational inequalities in Banach spaces. Math. Comput. Model. 54, 325-329 (2011)

24. Xu, HK: Inequalities in Banach spaces with applications. Nonlinear Anal. 16, 1127-1138 (1991)

25. Bruck, RE Jr.: Nonexpansive retracts of Banach spaces. Bull. Am. Math. Soc. 76, 384-386 (1970)

26. Takahashi, W, Toyoda, M: Weak convergence theorems for nonexpansive mappings and monotone mappings. J. Optim. Theory Appl. 118, 417-428 (2003)

27. Browder, FE: Nonlinear operators and nonlinear equations of evolution in Banach spaces. In: Nonlinear Functional Analysis (Proc. Sympos. Pure Math., vol. XVIII, Part 2, Chicago, III, 1968), pp. 1-308. Am. Math. Soc., Rhode Island (1976)

28. Xu, HK: Iterative algorithms for nonlinear operators. J. Lond. Math. Soc. 66, 240-256 (2002)

doi:10.1186/1029-242X-2013-76

Cite this article as: Wu et al.: A Korpelevich-like algorithm for variational inequalities. Journal of Inequalities and Applications 2013 2013:76.

\section{Submit your manuscript to a SpringerOpen ${ }^{\circ}$ journal and benefit from:}

- Convenient online submission

- Rigorous peer review

- Immediate publication on acceptance

Open access: articles freely available online

- High visibility within the field

- Retaining the copyright to your article 